\title{
Réviser les tarifs de cubage pour mieux gérer les forêts du Cameroun
}

Adeline FAYolLE Jacques RONDEUX Jean-Louis DouCET Gaëtan ERNST Christina Boulssou Samuel QueVAuvilLeRS Nils BOURLAND Richard FÉTÉKÉ Philippe Lejeune

Université de Liège Gembloux Agro-Bio Tech Unité gestion des ressources forestières et des milieux naturels Passage des Déportés, 2 5030 Gembloux Belgique

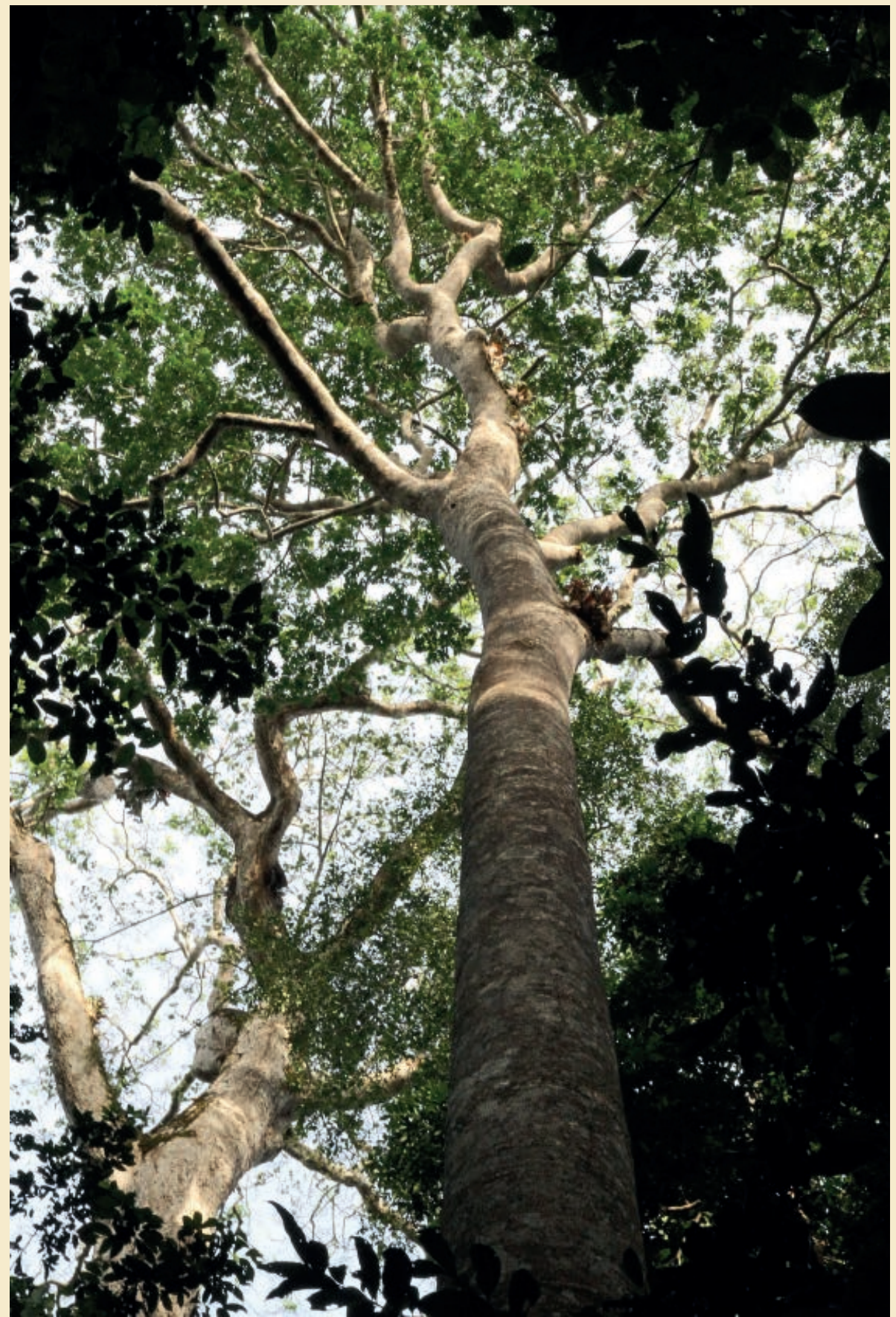

Photo 1.

Pied d'Entandrophragma cylindricum (sapelli). Photo J.-L. Doucet. 


\section{RÉSUMÉ}

\section{RÉVISER LES TARIFS DE CUBAGE POUR MIEUX GÉRER LES FORÊTS DU CAMEROUN}

L'utilisation des tarifs de cubage fait partie du quotidien des aménagistes et gestionnaires forestiers. Toutefois, elle se fait généralement au mépris des conditions d'application (zone géographique, gamme de diamètres), ce qui peut engendrer des erreurs dans les estimations de volume. L'objectif de cette étude est de tester la validité des tarifs de cubage actuellement disponibles pour trois essences des forêts du sud-est du Cameroun, dont ceux utilisés par l'administration nationale et, le cas échéant, de proposer des tarifs adaptés pour la zone d'étude. Trois espèces commerciales sont concernées : le sapelli, Entandrophragma cylindricum, le tali, Erythrophleum suaveolens, et l'assaméla, Pericopsis elata. Les données dendrométriques ont été collectées par échantillonnage destructif de 43 arbres régulièrement répartis sur toute la gamme de diamètres. Le volume du fût a été calculé par la méthode des billons successifs et des tarifs de cubage linéaires et non linéaires ont été ajustés aux données par la méthode des moindres carrés pondérés. Les résultats montrent que le meilleur modèle de cubage est non linéaire pour les trois espèces. Les équations utilisées jusqu'à présent par l'administration forestière sous-estiment significativement le volume des arbres et les erreurs d'estimation sont d'autant plus grandes que la taille de l'arbre est importante. Les tarifs de cubage proposés, une fois validés, devraient pouvoir à l'avenir être utilisés pour une meilleure estimation du volume des arbres dans la zone d'étude. Considérant les enjeux internationaux auxquels adhère le Cameroun (FLEGT et REDD+), il est crucial de disposer d'outils performants d'estimation du volume des arbres. Dans ce contexte, il s'avère important que l'administration forestière camerounaise puisse mener à bien un vaste programme de révision des tarifs de cubage.

Mots-clés : tarif de cubage, volume fût sur écorce, diamètre de référence, biais, erreur, Entandrophragma cylindricum, Erythrophleum suaveolens, Pericopsis elata, forêt semi-décidue, Cameroun.

\section{ABSTRACT}

\section{REVISING VOLUME TABLES FOR BETTER MANAGEMENT OF CAMEROON'S FORESTS}

Volume tables are routinely used by forest planners and managers, but generally with no regard for the conditions in which they are applied (geographical zone, diameter range), which can cause errors in estimations of timber volumes. The aim of this study is to test the validity of currently available volume tables, including those used by the government, for three commercial tree species in the forests of south-eastern Cameroon, and if required to propose tables suited to the study zone. The three species concerned are sapelli, Entandrophragma cylindricum, tali, Erythrophleum suaveolens, and assamela Pericopsis elata. Dendrometric data were collected by destructive sampling of 43 trees that were evenly distributed across the diameter range. Stemwood volume was calculated through successive butt-log measurements and linear and non-linear volume tables were adjusted by means of the weighted least-squares method. The results show that the best volume tables for all three species are non-linear. The equations used by the forests authority until now significantly under-estimate standing timber volumes and the estimation errors increase with the size of the trees. The proposed volume tables, once validated, should in future produce more accurate estimations of standing timber in the study zone. Given the international challenges involving Cameroon (FLEGT and REDD+), it is essential that it should have effective tools for estimating standing timber volumes. It is therefore important for Cameroon's forests authority to conduct a large-scale programme to revise its volume tables.

Keywords: volume table, over-bark butt log volume, reference diameter, bias, error, Entandrophragma cylindricum, Erythrophleum suaveolens, Pericopsis elata, semi-deciduous forest, Cameroon.

\section{REVISAR LAS TABLAS DE CUBICACIÓN PARA UN MEJOR MANEJO DE LOS BOSQUES DE CAMERÚN}

La utilización de tablas de cubicación forma parte de las actividades cotidianas de los responsables de la ordenación y manejo forestal. No obstante, dichas tablas suelen emplearse sin tener en cuenta las condiciones de aplicación (zona geográfica, clases de diámetro), lo que puede provocar errores en las estimaciones volumétricas. El objetivo de este estudio es probar la validez de las tablas de cubicación actualmente disponibles para tres especies de los bosques del sudeste de Camerún, incluyendo las tablas empleadas por la administración nacional, y, llegado el caso, proponer tablas adaptadas al área estudiada. Las tres especies comerciales concernidas son: el sapelli, Entandrophragma cylindricum, el tali, Erythrophleum suaveolens, y la assamela, Pericopsis elata. La recogida de datos dasométricos se efectuó por muestreo destructivo de 43 árboles regularmente distribuidos en las diferentes clases de diámetro. Se calculó el volumen de fuste por el método de trozas sucesivas y las tablas de cubicación lineales y no lineales se ajustaron a los datos mediante el método de mínimos cuadrados ponderados. Los resultados muestran que el mejor modelo de cubicación en las tres especies es el no lineal. Las ecuaciones actualmente empleadas por la administración forestal subestiman significativamente el volumen de los árboles y, cuanto mayor es el tamaño del árbol, tanto más importantes son los errores de estimación. Las tablas de cubicación propuestas, una vez validadas, deberían poder utilizarse para una mejor estimación del volumen de árboles en la zona estudiada. Teniendo en cuenta los envites internacionales que envuelven a Camerún (FLEGT y REDD+), es esencial contar con herramientas eficientes para estimar el volumen de los árboles. En este contexto, es importante que la administración forestal camerunesa pueda llevar a cabo un amplio programa de revisión de las tablas de cubicación.

Palabras clave: tabla de cubicación, volumen fuste con corteza, diámetro de referencia, sesgo, error, Entandrophragma cylindricum, Erythrophleum suaveolens, Pericopsis elata, bosque semideciduo, Camerún. 


\section{Introduction}

L'exploitation forestière en Afrique centrale se concentre sur un faible nombre d'espèces commerciales (DE WASSEIGE et al., 2009) qui sont paradoxalement encore mal connues (AMSALLEM et al., 2004). Il existe notamment très peu d'études dendrométriques portant sur les essences africaines alors que l'estimation du matériel ligneux, à partir de tarifs de cubage appropriés, est un préalable à l'aménagement des forêts naturelles. En effet, on estime qu'il existe actuellement des études dendrométriques pour seulement $3 \%$ des espèces d'arbres du Cameroun et seulement $1 \%$ des espèces du Congo (HENRY et al., 2011). Les propos de LANLY (1965) à ce sujet semblent donc toujours d'actualité : "Malheureusement ce problème ne semble pas avoir été étudié avec l'urgence qu'il mérite dans le domaine des forêts tropicales : le petit nombre des études faites sur cette question témoigne du peu d'intérêt qu'ont pu lui apporter les forestiers sans doute préoccupés par d'autres tâches plus absorbantes. »

Les études dendrométriques peuvent comprendre plusieurs composantes et viser plusieurs objectifs dont un des principaux est incontestablement la construction de tarifs de cubage. Ceux-ci permettent d'estimer le volume d'un arbre en fonction d'une ou plusieurs caractéristiques dendrométriques directement mesurables sur le terrain telles que le diamètre ou la hauteur des arbres (LANLY, 1965 ; CAILLIEZ, 1980 ; RONDEUX, 1999). Il existe différents types de tarifs de cubage en fonction du nombre d'entrées qu'ils comportent. Les principales entrées sont le diamètre de référence mesuré à hauteur de poitrine $(1,30 \mathrm{~m})$ ou à $30 \mathrm{~cm}$ audessus des déformations (contreforts, empattements ou cannelures) selon les conventions internationales de mesure (CAILLIEZ, 1980), qui peut être associé à des hauteurs totales ou partielles dans le cadre de tarifs à deux entrées. En règle générale, deux types d'équations reliant le volume au diamètre sont utilisés : des relations linéaires de type polynomial et des relations non linéaires de type exponentiel (LANLY, 1965 ; RONDEUX, 1999 ; PICARD et al., 2012). En toute rigueur, les tarifs de cubage sont valables uniquement pour l'essence, la gamme de diamètres et la zone géographique des arbres ayant servi à les construire (RONDEUX, 1999) et, en outre, ils devraient être systématiquement assortis des paramètres permettant de calculer les intervalles de confiance des estimations (PICARD et al., 2012).

Le sapelli, Entandrophragma cylindricum (Sprague) Sprague, et le tali, Erythrophleum ivorense A. Chev. et E. suaveolens (Guill., Perr.) Brenan, sont respectivement les deuxième et troisième essences les plus exploitées par le secteur formel au Cameroun, à raison de $16 \%$ et $7 \%$ des volumes commercialisés (DE WASSEIGE et al., 2009). L'assaméla, Pericopsis elata (Harms) Meeuwen, est une essence noble à forte valeur commerciale, mais exploitée suivant des conditions très strictes du fait de son statut d'espèce protégée par la Convention sur le commerce International des espèces de faune et de flore sauvages menacées d'extinction - Cites (BOURLAND et al., 2012a, b). D'après GlobAllomeTree ${ }^{1}$, plateforme Internet qui regroupe

${ }^{1} \mathrm{http}: / /$ www.globallometree.org.

${ }^{2}$ Agence canadienne de développement international. les équations allométriques disponibles pour les estimations de volume, de biomasse et de stock de carbone forestier à l'échelle mondiale, douze tarifs de cubage sont disponibles pour E. cylindricum en Afrique (HENRY et al. 2013) et ce, malgré sa large distribution couvrant toute la région guinéo-congolaise. Les travaux ont été menés en ordre dispersé au Cameroun, au Congo, en Côte d'Ivoire, au Gabon, au Ghana, au Nigeria et en République centrafricaine (Rca) (tableau I). Néanmoins, les métadonnées liées à la construction de ces tarifs de cubage (caractéristiques de l'échantillon, qualité d'ajustement et erreur résiduelle des modèles) sont rarement disponibles. D'après cette même plateforme, il existerait trois études pour E. ivorense et/ou E. suaveolens, au Gabon, au Nigeria et en Rca, ainsi qu'une seule étude au Ghana pour $P$. elata, regroupée avec Afzelia africana (doussié ; tableau I).

Au Cameroun, l'Acdi' , en appuyant le programme d'évaluation des ressources forestières nationales, a mené les premières phases de l'inventaire national entre 1982 et 1990, à l'issue desquelles les tarifs de cubage officiels ont été établis. L'Organisation des Nations unies pour l'alimentation et l'agriculture ( $\mathrm{FaO}$ ) a conduit un nouvel inventaire national en 2003-2004 et établi de nouveaux tarifs de cubage mais dans les zones non parcourues par l'inventaire précédent (FAO, 2007). Des tarifs de cubage ont donc été établis au Cameroun par espèce ou groupe d'espèces et par zone géographique, correspondant aux différentes phases de l'inventaire (figure 1 et tableau II). Ces tarifs sont inclus dans le logiciel de Traitement informatique appliqué à la modélisation des aménagements (Tiama) et doivent être utilisés lors de la réalisation des plans d'aménagement et

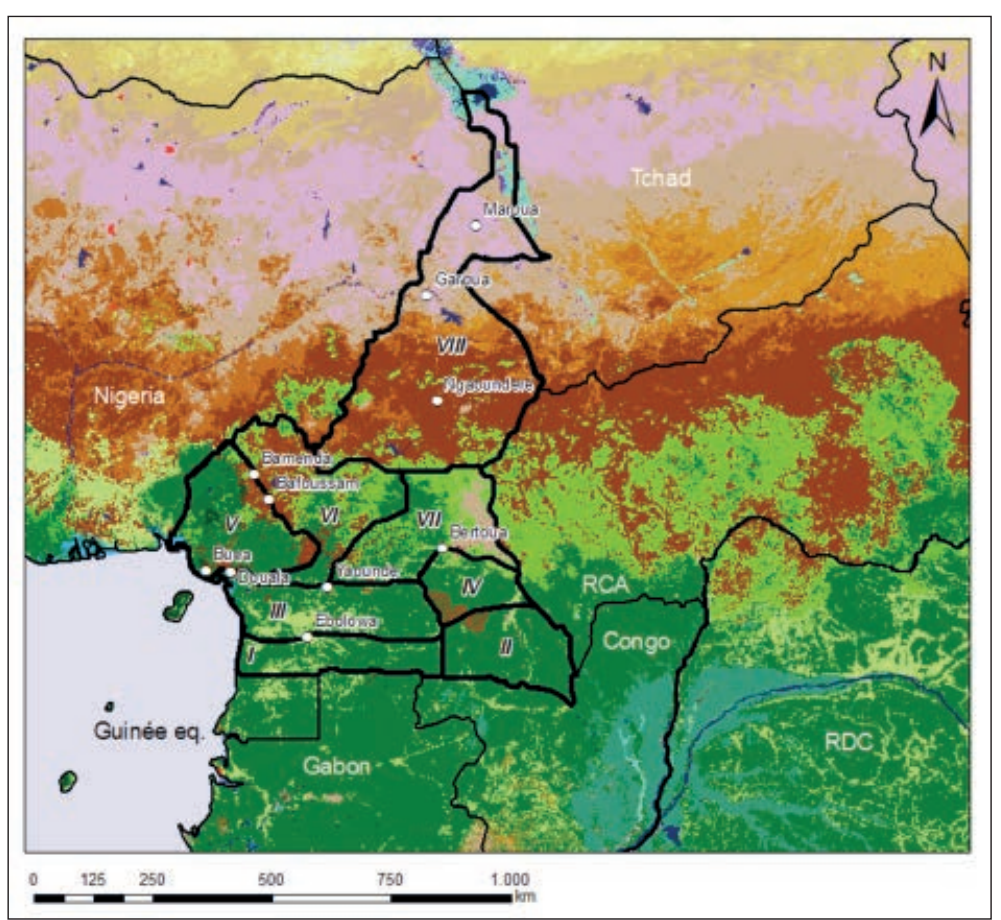

Figure 1.

Localisation de la zone d'étude (hachures rouges) et des différentes phases (I à VIII) de l'inventaire national des ressources forestières du Cameroun (FAO, 2007). La carte de végétation de MAYAUX et al. (2004) est présentée en fond en respectant le code couleur d'origine et les villes principales sont indiquées. 
lors de la planification des opérations d'exploitation. Ces équations sont malheureusement dépourvues d'informations relatives aux caractéristiques de l'échantillon, à la qualité de l'ajustement, et à l'erreur résiduelle du modèle. De plus, il existe assez souvent des différences significatives entre les volumes réels calculés après l'abattage et les volumes estimés par ces tarifs, ce qui amène à des controverses entre l'administration et les exploitants qui doivent payer des pénalités inhérentes à des dépassements de quota (TCHATAT et al., 2008).

Ainsi, l'objectif de ce travail a été, d'une part, de tester la validité des tarifs de cubage actuellement disponibles pour trois essences, le sapelli, le tali et l'assaméla, et, d'au- tre part, de proposer des tarifs adaptés au Sud-Est du Cameroun. Les tarifs de cubage ont été élaborés à partir des données dendrométriques obtenues par échantillonnage destructif de 43 arbres régulièrement répartis sur la gamme de diamètres. Ces tarifs sont assortis d'informations sur la zone géographique, l'effectif de l'échantillon, la gamme de diamètres, la qualité d'ajustement et l'erreur résiduelle des modèles. Les estimations fournies par ces tarifs de cubage ont ensuite été comparées avec celles des équations utilisées par l'administration forestière nationale et des autres équations existantes en Afrique.

Tableau I.

Tarifs de cubage à une et deux entrées, équations reliant le volume $(V)$ au diamètre de référence $(D)$ et/ou à la hauteur $(H)$, existants en Afrique pour les trois essences étudiées d'après GlobAllomeTree (http://www.globallometree.org ; HENRY et al., 2013), la plateforme Internet qui regroupe les équations allométriques disponibles pour les estimations de volume, de biomasse et de stock de carbone forestier à l'échelle mondiale. Les unités de chaque variable d'entrée $(D$ et $H)$ et de sortie sont données.

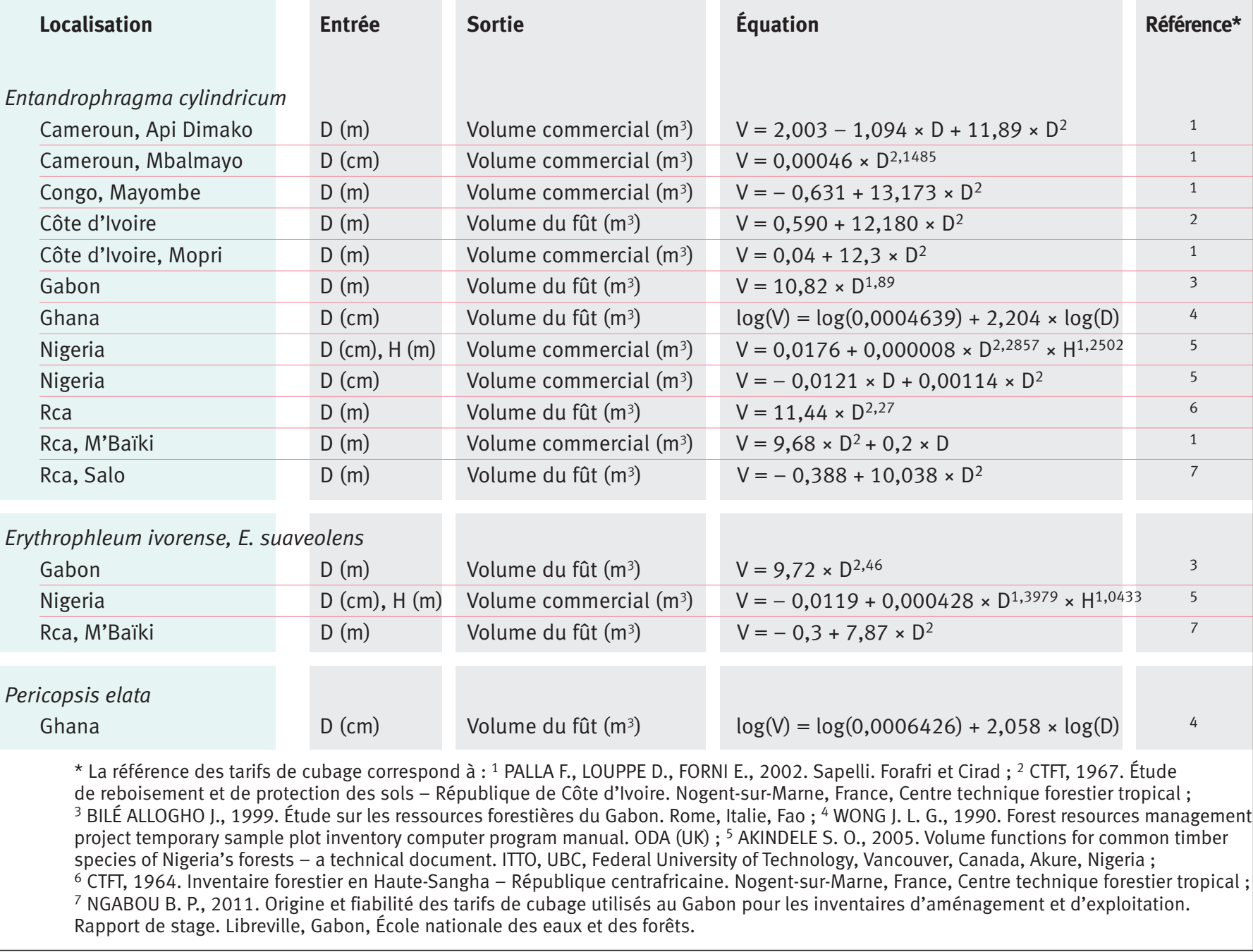


Tableau II.

Tarifs de cubage à une entrée reliant le volume du fût $\left(V\right.$, en $\left.\mathrm{m}^{3}\right)$ au diamètre de référence $(D$, en $\mathrm{cm})$ utilisés par l'administration camerounaise pour les trois essences étudiées (FAO, 2007). L'évaluation des ressources forestières nationales du Cameroun a été réalisée en différentes phases couvrant des zones géographiques différentes (figure 1). La zone d'étude se situe à cheval entre les phases II et III.

\begin{tabular}{l|l|l|}
\hline Espèce & Phase & Équation \\
\cline { 1 - 2 } & & \\
\hline Entandrophragma cylindricum & I & $\mathrm{V}=-0,97627+0,0287949 \times \mathrm{D}+0,0008307 \times \mathrm{D}^{2}$ \\
\hline Entandrophragma cylindricum & II & $\mathrm{V}=0,215-0,00009 \times \mathrm{D}+0,0009622 \times \mathrm{D}^{2}$ \\
\hline Entandrophragma cylindricum & III & $\mathrm{V}=0,000564 \times \mathrm{D}^{2,096088}$ \\
\hline Entandrophragma cylindricum & IV & $\mathrm{V}=0,000459 \times \mathrm{D}^{2,14853}$ \\
\hline Erythrophleum ivorense +E. suaveolens & I & $\mathrm{V}=-0,00676+0,0008579 \times \mathrm{D}^{2}$ \\
\hline Erythrophleum ivorense + E. suaveolens & II & $\mathrm{V}=1,858-0,03518 \times \mathrm{D}+0,0010283 \times \mathrm{D}^{2}$ \\
\hline Erythrophleum ivorense & III & $\mathrm{V}=0,000461 \times \mathrm{D}^{2,166608}$ \\
\hline Erythrophleum suaveolens & III & $\mathrm{V}=0,000672 \times \mathrm{D}^{2,07027}$ \\
\hline Erythrophleum ivorense & IV & $\mathrm{V}=0,000252 \times \mathrm{D}^{2,279235}$ \\
\hline Erythrophleum suaveolens & IV & $\mathrm{V}=0,000347 \times \mathrm{D}^{2,211969}$ \\
\hline Pericopsis elata & II & $\mathrm{V}=-0,609+0,0009668 \times \mathrm{D}^{2}$ \\
\hline Pericopsis elata & III & $\mathrm{V}=0,000519 \times \mathrm{D}^{2,161415}$ \\
\hline Pericopsis elata & IV & $\mathrm{V}=0,000186 \times \mathrm{D}^{2,388659}$ \\
\hline
\end{tabular}

\section{Matériel et méthodes}

\section{Zone d'étude}

La zone d'étude se situe dans le Sud-Est du Cameroun, dans le département du Haut-Nyong, plus précisément entre $3^{\circ} 01^{\prime}$ et $3^{\circ} 44^{\prime}$ de latitude Nord et entre $13^{\circ} 20^{\prime}$ et $14^{\circ} 31^{\prime}$ de longitude Est. L'étude a été conduite au niveau des différents chantiers d'exploitation de la société sous aménagement durable Pallisco. Les unités forestières d'aménagement (Ufa) exploitées par cette société sont localisées dans les phases II (Ufa 10030-10031) et IV (Ufa 10041-10042-10044) de l'inventaire national des ressources forestières du Cameroun (figure 1). Le climat équatorial chaud et humide est de type guinéen à quatre saisons. La grande saison des pluies s'étend de mi-août à mi-novembre, suivie de la grande saison sèche de minovembre à mi-mars, puis de la petite saison des pluies de mi-mars à juin et, enfin, de la petite saison sèche de juin à mi-août. La pluviosité varie entre 1500 et $2000 \mathrm{~mm}$ par an et la température moyenne annuelle oscille autour de $24^{\circ} \mathrm{C}$. La zone d'étude repose majoritairement sur une assise du précambrien inférieur. Le matériel parental est constitué de micaschistes au Nord et de grès quartzites au Sud. Les sols sont de type ferralitique rouge ou jaune, la topographie est vallonnée, l'altitude varie entre $600 \mathrm{~m}$ et $760 \mathrm{~m}$ et le réseau hydrographique s'avère dense (KOUADIO, DOUCET, 2009). La végétation relève de la forêt dense humide tropicale de transition entre les types sempervirent et semi-décidu (WHITE, 1983 ; LETOUZEY, 1985).

\section{Essences étudiées}

Le sapelli, Entandrophragma cylindricum (Meliaceae), est une espèce de la forêt dense humide à distribution omniguinéo-congolaise, particulièrement abondante dans les forêts semi-décidues. Sa distribution s'étend de la Sierra Leone à l'Ouganda, y compris dans les forêts du Mayombe au Sud (GERDAT, 1974). La base des arbres présente une très grande diversité morphologique, pouvant aller d'un cylindre presque parfait à une forme très empattée et/ou présentant des contreforts épais. Les arbres peuvent atteindre une quarantaine de mètres de hauteur, et jusqu'à 2,50 m de diamètre (photo 1 ). Le diamètre minimum d'exploitation (Dme) fixé par l'administration camerounaise est de $1 \mathrm{~m}$. La vitesse de croissance en diamètre est de $0,53 \pm 0,07 \mathrm{~cm}$ par an $(n=197)$ sur la zone d'étude (R. FÉTÉKÉ, com. pers.) et la densité du bois est comprise entre 0,50 et $0,63 \mathrm{~g} / \mathrm{cm}^{3}$ (ZANNE et al., 2009). Le bois du sapelli est généralement utilisé en placage pour meuble, agencement ou décoration. Cette espèce, abondante localement, est classée comme vulnérable dans la liste rouge de l'lucn.

Le tali, Erythrophleum suaveolens (Fabaceae), est une espèce des forêts denses humides sempervirentes et semidécidues à distribution guinéo-congolaise qui s'étend de la Sierra Leone à la République démocratique du Congo (GERDAT, 1977). Son aire de distribution chevauche celle d'Erythrophleum ivorense dans la partie ouest mais seule E. suaveolens est présente dans la zone d'étude (DUMINIL et al., 2010). Erythrophleum suaveolens est un grand arbre ayant des contreforts épais mais peu étendus. Il peut atteindre une quarantaine de mètres de hauteur totale, et plus de 


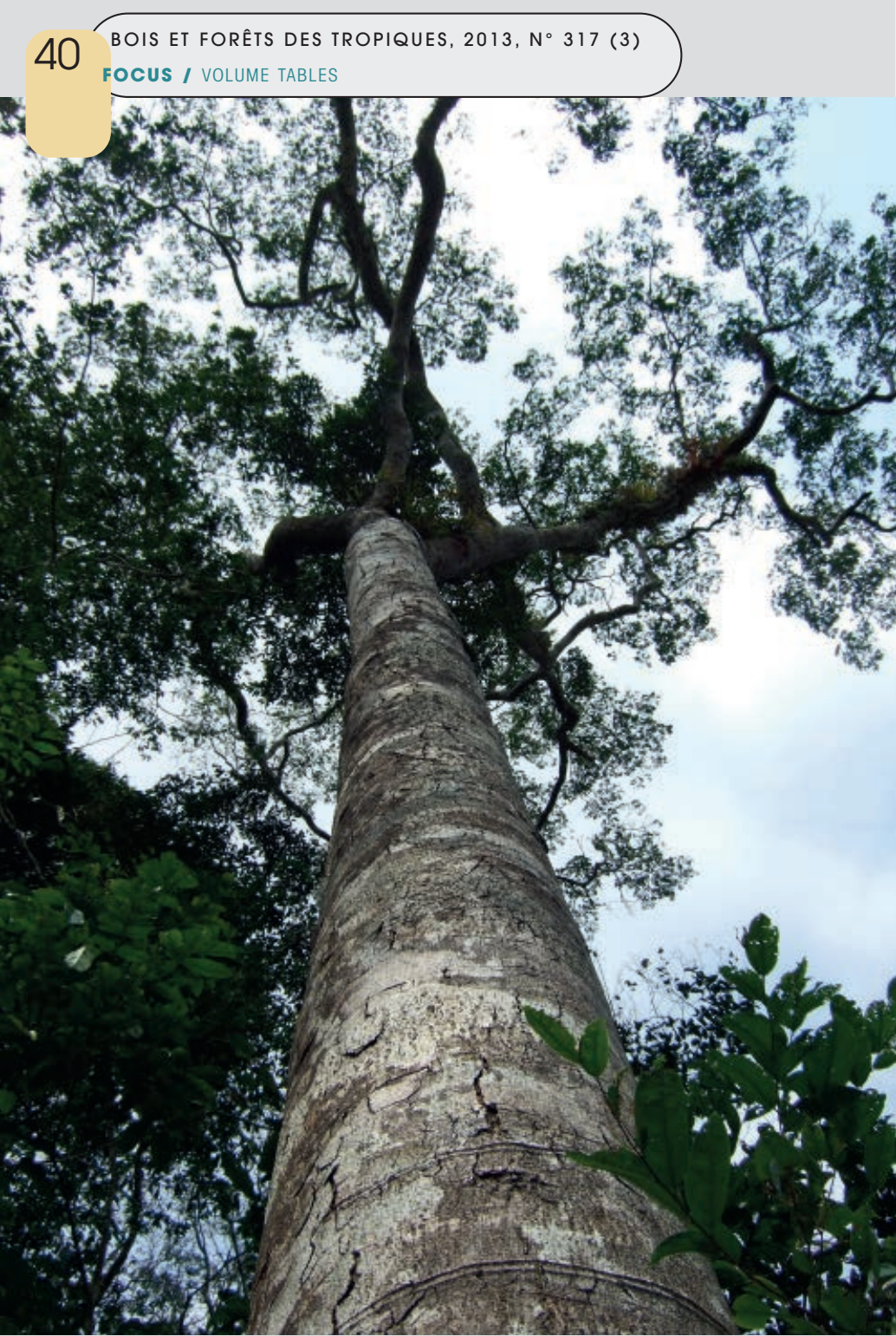

Photo 2.

Pied d'Erythrophleum suaveolens (tali).

Photo N. Bourland.

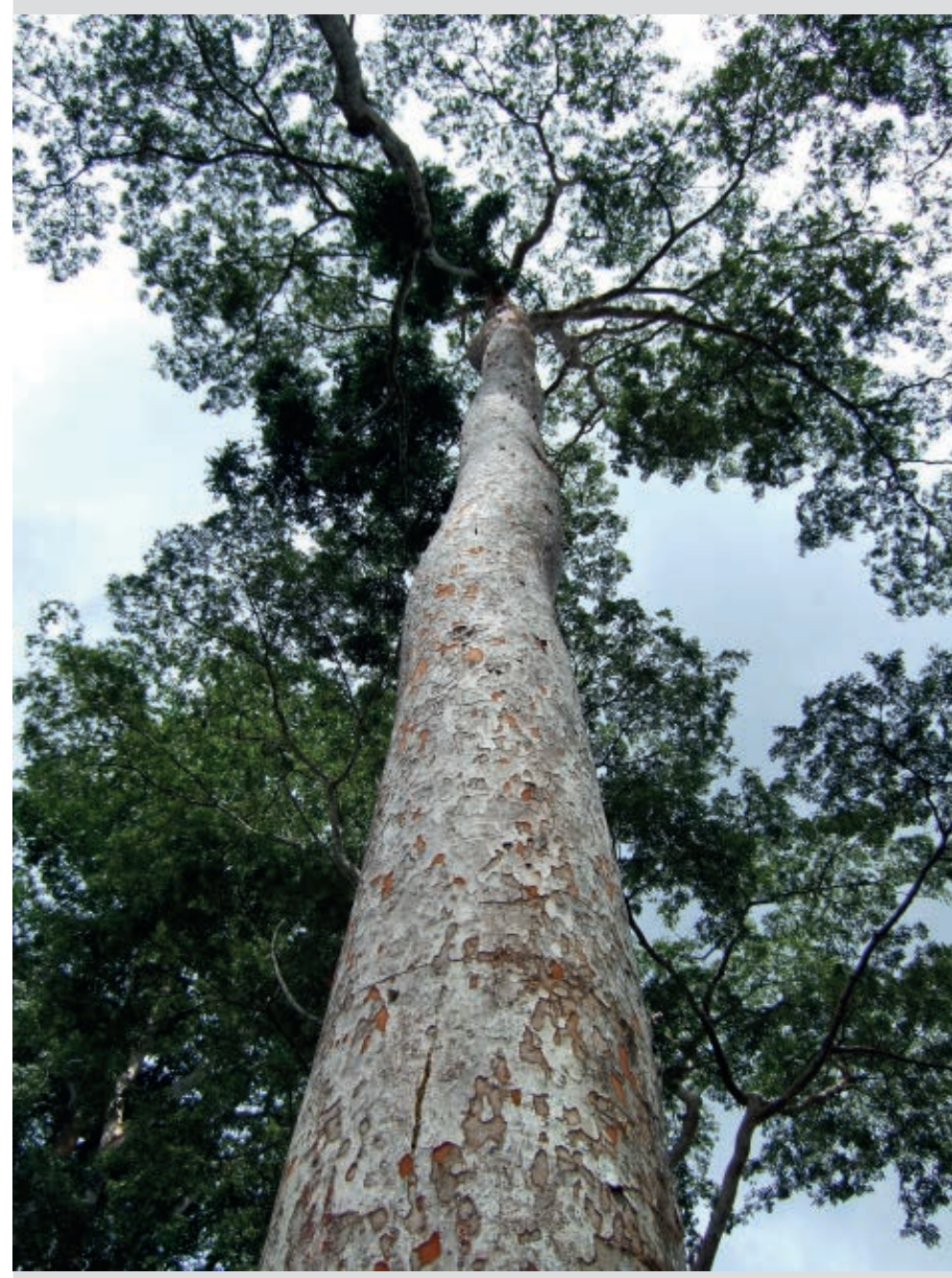

Photo 3.

Pied de Pericopsis elata (assaméla).

Photo N. Bourland.
$1 \mathrm{~m}$ de diamètre. Le fût est souvent sinueux (photo 2). Le Dme est de 0,5 m au Cameroun. La vitesse de croissance en diamètre est de $0,68 \pm 0,10 \mathrm{~cm}$ par an $(n=38)$ sur la zone d'étude (KOUADIO, 2009) et la densité du bois est comprise entre 0,75 et $0,98 \mathrm{~g} / \mathrm{cm}^{3}$ pour l'Afrique (ZANNE et al., 2009). Le bois du tali est généralement utilisé en menuiserie lourde extérieure, parquet, traverse hydraulique, ou charpente. Cette espèce, abondante localement, ne dispose pas de statut particulier en termes de conservation.

L'assaméla, Pericopsis elata (Fabaceae), est une espèce des forêts denses humides semi-décidues présentant une distribution particulièrement discontinue (HAWTHORNE, 1995). Il s'agit d'un arbre dominant qui peut facilement atteindre 40 à $50 \mathrm{~m}$ de haut mais qui excède rarement $1,50 \mathrm{~m}$ de diamètre. Le fût est tortueux à droit suivant le stade de développement, et présente généralement des cannelures à la base (photo 3). Le Dme est fixé à $0,9 \mathrm{~m}$ suite à une circulaire de juin 2010, il était de $1 \mathrm{~m}$ avant cette date. La croissance annuelle en diamètre est de 0,31 \pm
$0,05 \mathrm{~cm}$ par an $(\mathrm{n}=143)$ sur la zone d'étude (BOURLAND et al., 2012a) et la densité du bois est comprise entre 0,57 et $0,71 \mathrm{~g} / \mathrm{cm}^{3}$ (ZANNE et al., 2009). S'agissant des caractéristiques du bois, le duramen de l'assaméla possède de très bonnes propriétés technologiques et une bonne durabilité naturelle. Cette espèce figure dans l'annexe II de la Cites, espèces dont le commerce des spécimens doit être réglementé pour éviter une exploitation incompatible avec leur survie, et elle est classée en danger d'extinction sur la liste rouge de l'lucn.

\section{Protocole expérimental}

Dans le cadre de cette étude, des données dendrométriques ont été obtenues par échantillonnage destructif de 43 arbres, répartis comme suit : 15 pieds d'Entandrophragma cylindricum, 14 pieds d'Erythrophleum suaveolens et 14 pieds de Pericopsis elata, uniformément répartis sur l'ensemble de la gamme de diamètres pour chacune des 
Tableau III.

Données dendrométriques ayant servi à construire les tarifs de cubage proposés dans la présente étude et à tester la validité des équations utilisées par l'administration pour les trois essences étudiées. Le diamètre de référence $(D$, en $\mathrm{m})$, la hauteur de référence ( $h$, en $\mathrm{m}$ ), la longueur de la grume ( $L$, en $\mathrm{m})$ et le volume du fût $\left(V\right.$, en $\left.\mathrm{m}^{3}\right)$ sont donnés pour chaque arbre.

\begin{tabular}{|c|c|c|c|}
\hline D & h & $\mathbf{L}$ & V \\
\hline \multicolumn{4}{|c|}{ Entandrophragma cylindricum } \\
\hline 0,375 & 1,30 & 11,79 & 1,08 \\
\hline 0,385 & 1,30 & 16,78 & 1,23 \\
\hline 0,401 & 1,30 & 20,20 & 1,54 \\
\hline 0,480 & 1,30 & 22,22 & 2,12 \\
\hline 0,537 & 1,30 & 19,66 & 2,54 \\
\hline 0,740 & 3,12 & 39,42 & 10,65 \\
\hline 0,950 & 3,03 & 23,59 & 13,06 \\
\hline 0,963 & 1,30 & 26,89 & 12,22 \\
\hline 1,020 & 1,53 & 23,95 & 13,02 \\
\hline 1,091 & 1,78 & 22,86 & 14,84 \\
\hline 1,297 & 6,72 & 30,12 & 32,66 \\
\hline 1,417 & 2,98 & 19,20 & 24,17 \\
\hline 1,477 & 1,30 & 16,45 & 16,47 \\
\hline 1,611 & 4,13 & 23,03 & 32,70 \\
\hline 1,925 & 5,02 & 16,89 & 47,52 \\
\hline \multicolumn{4}{|c|}{ Erythrophleum suaveolens } \\
\hline 0,503 & 1,30 & 20,97 & 2,71 \\
\hline 0,535 & 1,30 & 14,25 & 2,77 \\
\hline 0,572 & 4,16 & 14,67 & 3,17 \\
\hline 0,612 & 3,75 & 18,35 & 4,10 \\
\hline 0,782 & 2,66 & 21,15 & 6,01 \\
\hline 0,809 & 3,14 & 18,38 & 7,08 \\
\hline 0,811 & 2,21 & 15,20 & 6,32 \\
\hline 0,918 & 2,44 & 16,16 & 8,66 \\
\hline 1,007 & 3,12 & 20,28 & 13,15 \\
\hline 1,062 & 2,52 & 17,96 & 12,22 \\
\hline 1,138 & 4,04 & 12,94 & 11,60 \\
\hline 1,210 & 2,24 & 17,65 & 16,70 \\
\hline 1,242 & 2,09 & 12,13 & 14,26 \\
\hline 1,375 & 4,24 & 23,23 & 26,97 \\
\hline \multicolumn{4}{|c|}{ Pericopsis elata } \\
\hline 0,223 & 1,30 & 16,21 & 0,42 \\
\hline 0,281 & 1,30 & 11,50 & 0,55 \\
\hline 0,344 & 1,30 & 22,83 & 1,37 \\
\hline 0,507 & 2,32 & 23,87 & 2,70 \\
\hline 0,520 & 1,30 & 24,13 & 3,32 \\
\hline 0,548 & 1,30 & 22,12 & 4,49 \\
\hline 0,625 & 1,30 & 24,95 & 4,33 \\
\hline 0,666 & 1,30 & 19,25 & 4,74 \\
\hline 0,672 & 2,76 & 22,76 & 5,63 \\
\hline 0,816 & 2,95 & 23,65 & 9,00 \\
\hline 0,932 & 2,08 & 21,91 & 9,71 \\
\hline 1,012 & 1,86 & 22,38 & 14,69 \\
\hline 1,020 & 2,60 & 25,16 & 15,63 \\
\hline 1,075 & 1,90 & 17,87 & 12,29 \\
\hline
\end{tabular}

trois essences (tableau III et figure 2). Afin de respecter la législation camerounaise qui interdit l'abattage des arbres en dessous du Dme, les arbres ayant un diamètre de référence inférieur au Dme ont été prélevés lors de l'ouverture des routes dans une bande de cinq mètres de part et d'autre de la voirie. Cette bande est quasi totalement rasée pour l'ensoleillement des pistes, mais par endroits quelques arbres sont laissés sur pied pour faciliter le passage des animaux arboricoles (pont de singes). Présents dans une zone auparavant inexploitée, ces arbres n'ont pas connu de conditions de croissance particulières. Quant aux arbres ayant un diamètre supérieur ou égal au Dme, ceux-ci ont été abattus au sein du chantier d'exploitation et mesurés avant le débardage.

Le volume du fût $\left(V\right.$ en $\left.\mathrm{m}^{3}\right)$ a été estimé par la méthode des billons successifs et le volume de chaque billon de deux mètres a été déterminé par la formule du tronc de cône (RONDEUX, 1999). Avant l'abattage, le diamètre de référence $(D$, en $\mathrm{m})$ a été mesuré au ruban diamétrique à $1,30 \mathrm{~m}$ du niveau du sol ou, le cas échéant, à $30 \mathrm{~cm}$ au-dessus des déformations (CAILLIEZ, 1980). Après l'abattage, une chevillière suédoise a été déroulée le long de la bille afin de matérialiser à la craie les différents niveaux de mesure du diamètre, lesquels correspondent à des billons successifs de deux mètres de longueur chacun, et ce jusqu'à la déformation due à la première grosse branche. Le dernier diamètre a été mesuré $30 \mathrm{~cm}$ avant la zone de déformation et la longueur du billon a été notée (diamètres toujours mesurés au ruban diamétrique).
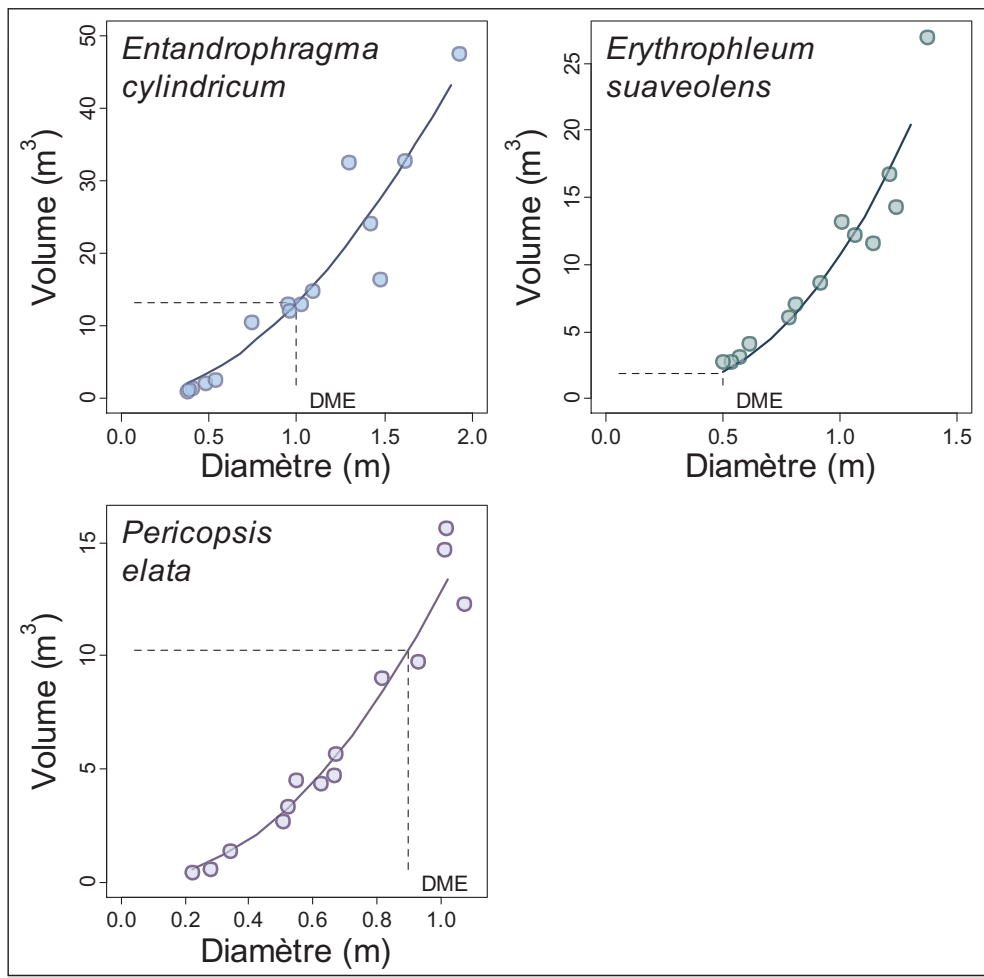

Figure 2.

Relation entre le volume du fût (en $\mathrm{m}^{3}$ ) et le diamètre (en $\mathrm{m}$ ) pour les trois espèces étudiées : Entandrophragma cylindricum, Erythrophleum suaveolens et Pericopsis elata. La courbe correspond à l'ajustement du meilleur modèle, le modèle non linéaire (M4), pour les trois espèces (tableau III). Le Dme et le volume du fût au Dme sont indiqués en pointillés. 


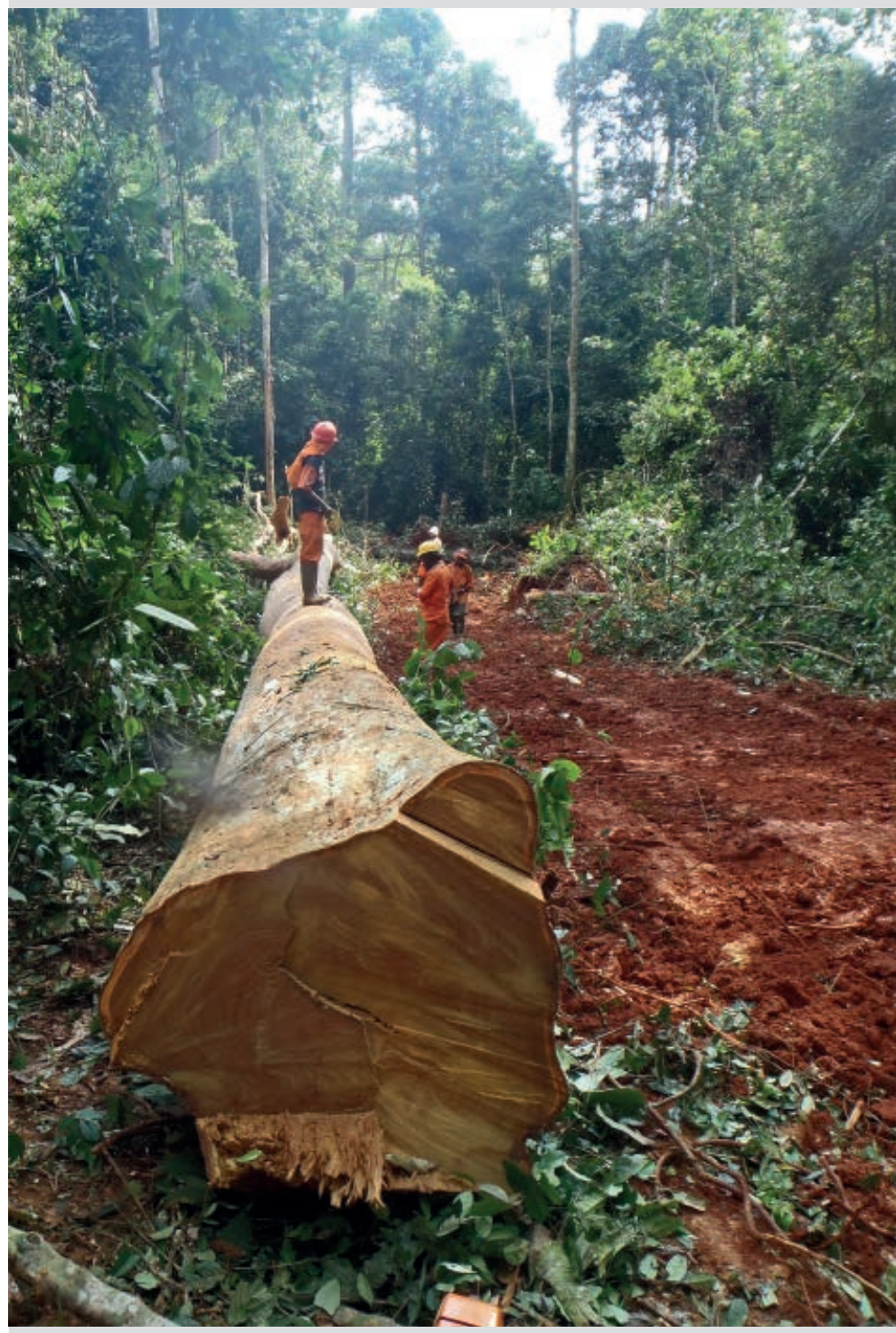

Photo 4.

Fût de Pericopsis elata (assaméla). Photo C. Bouissou.

L'ensemble des analyses statistiques a été réalisé avec le logiciel libre R (R DEVELOPMENT CORE TEAM, 2013). Les modèles linéaires de tarif de cubage ont été ajustés à l'aide de la fonction $l m$. Les modèles non linéaires de tarif de cubage ont été ajustés avec la fonction nls en utilisant les paramètres de la transformation logarithmique du modèle $M 4, \log (V)=\log (a)+b \times \log (D)$, comme paramètres d'entrée de la régression non linéaire. L'ajustement non linéaire a été préféré à l'ajustement linéaire via la transformation logarithmique car il permet de comparer directement les critères d'ajustement avec ceux des modèles linéaires (M1, M2, M3), la même variable étant modélisée ( $V$ et non $\log (V))$. Les régressions non paramétriques ont été ajustées à l'aide de la fonction lowess. paramétrique a été ajustée à ces données afin de visualiser graphiquement les tendances à une augmentation ou diminution de l'erreur en fonction de la taille de l'arbre. 


\section{Résultats}

\section{Volume par billons successifs}

En tout, 15 arbres ont été échantillonnés pour Entandrophragma cylindricum. Le diamètre de référence et la longueur de la grume varient de 0,375 à 1,925 m, et de 11,79 à $39,42 \mathrm{~m}$, respectivement (tableau III). Le volume moyen est de $14,1 \mathrm{~m}^{3}$ et le volume maximum est de $47,5 \mathrm{~m}^{3}$. Ce sont 14 arbres qui ont été échantillonnés pour Erythrophleum suaveolens. Le diamètre de référence et la longueur de la grume varient de 0,503 à 1,375 m, et de 12,13 à 23,23 m, respectivement. Le volume moyen est de $9,7 \mathrm{~m}^{3}$ et le volume maximum est de $27,0 \mathrm{~m}^{3}$. Enfin, 14 arbres ont été échantillonnés pour Pericopsis elata. Le diamètre de référence et la longueur de la grume varient de 0,223 à $1,075 \mathrm{~m}$, et de 11,50 à $25,16 \mathrm{~m}$, respectivement. Le volume moyen est de $6,3 \mathrm{~m}^{3}$ et le volume maximum est de $15,6 \mathrm{~m}^{3}$.

\section{Tarifs de cubage}

Des tarifs de cubage à une entrée ont été établis pour les trois essences étudiées (tableau IV et figure 2). Pour les trois essences, le meilleur modèle est de loin le modèle non linéaire M4 (AIC le plus faible). Il faut cependant noter que les trois modèles linéaires ( $M 1, M 2$, et $M 3)$ s'ajustent relativement bien aux données avec un coefficient de détermination supérieur à $0,907,0,892$ et 0,915 et un écart-type résiduel inférieur à 3,784, 2,202 et 2,170 $\mathrm{m}^{3}$, respectivement pour E. cylindricum, E. suaveolens et $P$. elata.

\section{Qualité des estimations}

La qualité des estimations des tarifs de cubage disponibles pour les trois essences étudiées, y compris les tarifs établis dans le cadre de cette étude (modèle M4 pour les trois essences), a été évaluée. En ce qui concerne Entandrophragma cylindricum, espèce pour laquelle plusieurs tarifs sont disponibles en Afrique, les estimations de

\section{Tableau IV.}

Ajustement de tarifs de cubage à une entrée reliant le volume du fût $\left(V\right.$, en $\left.\mathrm{m}^{3}\right)$ au diamètre de référence $(D$, en $\mathrm{m}$ ) pour les trois essences étudiées. L'estimation et l'intervalle de confiance (entre crochets) à $95 \%$ est donné pour chacun des paramètres $a, b$, et $c$. Le coefficient de détermination $\left(R^{2}\right)$, l'écart-type résiduel $(R S E)$ et le critère d'Akaike $(A / C)$ sont donnés pour chaque équation. Le meilleur modèle selon les critères d'ajustement utilisés est indiqué en gras pour chaque espèce.

\begin{tabular}{|c|c|c|c|c|c|c|}
\hline \multirow[t]{2}{*}{ Modèle } & \multicolumn{3}{|c|}{ Paramètres [intervalle de confiance à 95 \%] } & \multirow[t]{2}{*}{$R^{2}$} & \multirow[t]{2}{*}{ RSE } & \multirow[t]{2}{*}{$A I C$} \\
\hline & a & b & c & & & \\
\hline \multicolumn{7}{|c|}{ Entandrophragma cylindricum } \\
\hline M1 & $\begin{array}{c}-8,432 \\
{[-11,26 ;-5,6]}\end{array}$ & $\begin{array}{c}23,740 \\
{[19,36 ; 28,12]}\end{array}$ & & 0,907 & 3,784 & 81,6 \\
\hline M2 & $\begin{array}{c}-0,465 \\
{[-2,1 ; 1,17]}\end{array}$ & $\begin{array}{c}13,115 \\
{[10,79 ; 15,44]}\end{array}$ & & 0,913 & 3,647 & 80,5 \\
\hline M3 & $\begin{array}{c}-3,999 \\
{[-10,84 ; 2,85]}\end{array}$ & $\begin{array}{c}10,341 \\
{[-9,11 ; 29,79]}\end{array}$ & $\begin{array}{c}7,556 \\
{[-3,15 ; 18,27]}\end{array}$ & 0,916 & 3,600 & 80,9 \\
\hline M4 & $\begin{array}{c}12,724 \\
{[10,33 ; 15]}\end{array}$ & $\begin{array}{c}2,000 \\
{[1,64 ; 2,44]}\end{array}$ & & 0,898 & 3,699 & 17,0 \\
\hline \multicolumn{7}{|c|}{ Erythrophleum suaveolens } \\
\hline M1 & $\begin{array}{c}-8,057 \\
{[-11,22 ;-4,89]}\end{array}$ & $\begin{array}{c}19,651 \\
{[15,54 ; 23,76]}\end{array}$ & & 0,892 & 2,202 & 61,3 \\
\hline M2 & $\begin{array}{c}-0,619 \\
{[-1,97 ; 0,73]}\end{array}$ & $\begin{array}{c}11,700 \\
{[9,84 ; 13,56]}\end{array}$ & & 0,935 & 1,714 & 54,3 \\
\hline M3 & $\begin{array}{c}4,652 \\
{[-4,61 ; 13,91]}\end{array}$ & $\begin{array}{c}-13,548 \\
{[-37,09 ; 10]}\end{array}$ & $\begin{array}{c}19,525 \\
{[5,8 ; 33,25]}\end{array}$ & 0,938 & 1,673 & 54,4 \\
\hline M4 & $\begin{array}{c}10,920 \\
{[9,88 ; 11,95]}\end{array}$ & $\begin{array}{c}2,248 \\
{[1,88 ; 2,66]}\end{array}$ & & 0,919 & 1,647 & $-7,1$ \\
\hline \multicolumn{7}{|c|}{ Pericopsis elata } \\
\hline M1 & $\begin{array}{c}-3,391 \\
{[-4,63 ;-2,16]}\end{array}$ & $\begin{array}{c}14,470 \\
{[11,81 ; 17,13]}\end{array}$ & & 0,915 & 2,170 & 50,8 \\
\hline M2 & $\begin{array}{c}-0,301 \\
{[-0,78 ; 0,18]}\end{array}$ & $\begin{array}{c}13,082 \\
{[11,64 ; 14,52]}\end{array}$ & & 0,968 & 1,337 & 37,3 \\
\hline M3 & $\begin{array}{c}-0,220 \\
{[-2,05 ; 1,61]}\end{array}$ & $\begin{array}{c}-0,365 \\
{[-8,25 ; 7,52]}\end{array}$ & $\begin{array}{c}13,395 \\
{[6,45 ; 20,34]}\end{array}$ & 0,965 & 1,396 & 39,3 \\
\hline M4 & $\begin{array}{c}12,883 \\
{[11,55 ; 14,22]}\end{array}$ & $\begin{array}{c}2,157 \\
{[1,87 ; 2,48]}\end{array}$ & & 0,945 & 1,361 & $-139,4$ \\
\hline
\end{tabular}


volume du modèle $M 4$, et celles des équations développées dans le cadre du projet d'Aménagement pilote intégré (Api) à Dimako (Cameroun), au Mayombe (Congo), en Côte d'Ivoire (y compris à Mopri), au Gabon, au Ghana et en République centrafricaine ne sont pas biaisées (tableau V). La pente de la régression linéaire entre les données de volume observées et estimées par ces tarifs est proche de 1 bien que l'ordonnée à l'origine soit différente de 0 . L'EF est importante et proche de la valeur du $R^{2}$ de la régression linéaire pour ces tarifs. Le RMSE et l'erreur relative sont relativement importants $\left(>4 \mathrm{~m}^{3}\right.$ et entre $-1,6 \%$ et $44,3 \%$, respectivement) mais les erreurs d'estimation sont uniformément réparties autour de 0 (figure $3 a, f, h-l$ et $n$ ). Deux arbres se distinguent cependant par l'erreur importante qui est faite dans l'estimation de leur volume. Le premier se caractérise par une sous-estimation importante, il s'agit

\begin{abstract}
Tableau V.
Qualité des estimations des tarifs de cubage établis dans le cadre de cette étude (tableau IV), des équations utilisées par l'administration camerounaise (tableau II) et des équations à une entrée existantes en Afrique (tableau I) pour les trois essences étudiées. Les résultats du test de Student sur données appariées (statistique $t$ et valeur de probabilité $P$ ) et de la régression linéaire (ordonnée à l'origine, pente et $R^{2}$ ) entre les données de volume observées et les prédictions, ainsi que l'efficacité de la modélisation ( $E F)$ sont donnés pour chaque modèle de même que l'erreur quadratique moyenne (RMSE, en $\mathrm{m}^{3}$ ) et l'erreur relative moyenne (\%). Les équations donnant des estimations non biaisées $(P>0,05)$ sont indiquées en gras.
\end{abstract}

\section{Équation}

Entandrophragma cylindricum $(\mathrm{n}=15)$

a) Cameroun, M4

b) Cameroun, phase I

c) Cameroun, phase II

d) Cameroun, phase III

e) Cameroun, phase IV

f) Cameroun, Api Dimako

g) Cameroun, Mbalmayo

h) Congo, Mayombe

i) Côte d'Ivoire

j) Côte d'Ivoire, Mopri

k) Gabon

l) Ghana

m) Nigeria

n) $\mathbf{R c a}$

o) Rca, M'Baïki

p) Rca, Salo

Erythrophleum suaveolens $(n=14)$

a) Cameroun, M4

b) Cameroun, phase I (E. ivo + E. sua)

c) Cameroun, phase II (E. ivo + E. sua)

d) Cameroun, phase III (E. ivo)

e) Cameroun, phase III (E. sua)

f) Cameroun, phase IV (E. ivo)

g) Cameroun, phase IV (E. sua)

h) Gabon

i) Rca, M’Baïki

Pericopsis elata $(n=14)$

\section{a) Cameroun, M4}

b) Cameroun, phase II

c) Cameroun, phase III

d) Cameroun, phase IV

e) Ghana t $\quad P$

0,00

2,49

2,53

3,10

2,90

0,04

2,89

0,08

0,05

0,40

2,01

0,15

2,25

0,35

2,53

2,72

2,72

0,00

2,84

2,53

1,58

2,27

2,69

2,51

1,96

3,70

0,997

0,014

0,025

0,138

0,041

0,019

0,026

$\mathbf{0 , 0 7 2}$

0,003

0,08

4,36

2,62

3,07

3,78

0,024

0,008

0,012

0,012

0,934

0,693

0,064

0,883

$\mathbf{0 , 7 2 8}$

0,024

0,017

$\mathbf{0 , 9 4 1}$

0,001

0,009

0,002
Ord.

Pente

$R^{2}$

EF

RMSE

Erreur (\%)

$0,999 \quad 0,41$

0,02

0,14

0,95

1,23

$-1,24$

1,23

1,00

$-0,19$

0,37

$-0,27$

1,51

1,02

1,83

0,29

0,89

0,97

1,29

1,29

1,32

1,23

1,09

1,23

0,94

1,02

1,01

1,23

0,91

1,14

0,90

1,27

1,23

1,23

$\mathbf{0 , 8 9}$

0,893

0,890

0,888

0,887

$\mathbf{0 , 8 8 9}$

0,887

0,890

0,890

0,890

0,892

$\mathbf{0 , 8 8 6}$

0,889

$\mathbf{0 , 8 8 4}$

0,891

0,890

0,890

1,02

1,44

0,913

0,905

$-1,21$

$-1,80$

1,48

0,912

1,16

1,29

$\mathbf{0 , 9 1 0}$

0,907

0,914

0,912

1,23

$-0,35$

$\mathbf{0 , 4 7}$

$\mathbf{1 , 0 6}$

$\mathbf{0 , 9 1 8}$

$-0,75$

1,57

0,905

0,06

0,52

0,99

1,35

0,940

0,941

1,17

0,940

0,53

1,11

0,936

1,54

0,941

\begin{tabular}{l}
\hline $\mathbf{0 , 8 9 7}$ \\
0,791 \\
0,787 \\
\hline 0,736 \\
0,781 \\
\hline $\mathbf{0 , 8 9 2}$ \\
\hline 0,783 \\
\hline $\mathbf{0 , 8 9 4}$ \\
\hline $\mathbf{0 , 8 9 8}$ \\
\hline $\mathbf{0 , 8 9 7}$ \\
\hline $\mathbf{0 , 8 3 0}$ \\
\hline $\mathbf{0 , 8 8 5}$ \\
\hline 0,841 \\
\hline $\mathbf{0 , 8 8 1}$ \\
\hline 0,794 \\
\hline 0,795
\end{tabular}

4,29

15,2

6,12

$-11,1$

6,18

$-7,5$

6,88

$-22,1$

6,26

$-20,2$

4,41

44,3

6,24

$-20,0$

4,35

3,2

4,28

25,4

4,30

12,4

5,53

0,8

4,54

3,2

5,33

$-13,0$

4,62

$-1,6$

6,08

$-9,6$

6,06

$-19,0$

0,795

$\mathbf{0 , 9 1 9}$

1,85

0,1

0,718

3,46

$-18,4$

0,734

3,36

$-13,4$

$\mathbf{0 , 8 8 0}$

2,26

$-7,9$

0,815

2,80

$-12,5$

0,833

2,66

$-16,8$

0,831

$\mathbf{0 , 8 9 9}$

2,68

$-15,1$

$\mathbf{2 , 0 7}$

$-13,3$

0,571

4,27

$-29,9$

$-0,16$

\begin{tabular}{|r|r|r|}
\hline $\mathbf{0 , 9 4 5}$ & $\mathbf{1 , 1 5}$ & $\mathbf{4 , 7}$ \\
\hline 0,707 & 2,65 & $-43,6$ \\
\hline 0,886 & 1,66 & $-11,1$ \\
\hline 0,884 & 1,67 & $-19,4$ \\
\hline 0,639 & 2,94 & $-27,6$ \\
\hline
\end{tabular}

- 

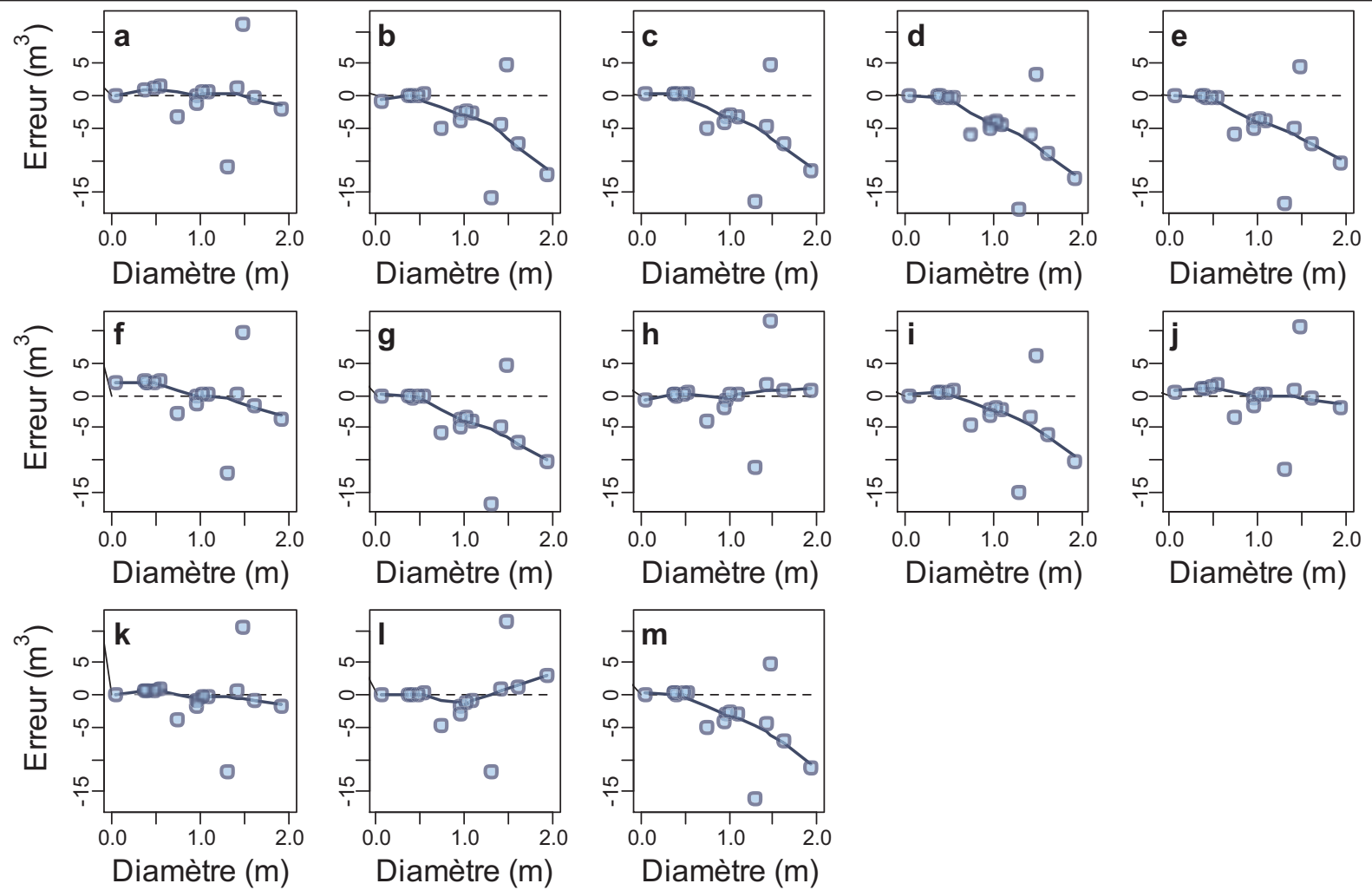

Figure 3.

Entandrophragma cylindricum : erreur d'estimation du tarif de cubage établi dans le cadre de cette étude (a), des équations utilisées par l'administration camerounaise (b-e pour les phases d'inventaire I-IV) et des autres équations existantes au Cameroun, Api (f), Cameroun, Mbalamayo (g), Congo, Mayombe (h), Gabon (i), en Côte d'Ivoire (j), Côte d'Ivoire, Mopri (k), en Rca (l) et en Rca, M'Baïki $(\mathrm{m})$ pour Entandrophragma cylindricum en fonction du diamètre (en $\mathrm{m}$ ). La courbe correspond à l'ajustement de la régression non paramétrique. Une valeur nulle indique une adéquation parfaite entre l'estimation du volume et le volume obtenu par la méthode des billons successifs. Les valeurs négatives et positives indiquent les sous- et sur estimations, respectivement.

d'un arbre de 1,3 $\mathrm{m}$ de diamètre particulièrement élancé et présentant un fût de 30,12 m de long. Le second se caractérise par une surestimation importante ; il s'agit d'un arbre de 1,48 $\mathrm{m}$ de diamètre présentant un fût de seulement $16,45 \mathrm{~m}$ de long. Les équations utilisées par l'administration camerounaise dans les phases d'inventaire I à IV, de Mbalamayo (Cameroun), de M'Baïki et de Salo (Rca) sousestiment significativement les volumes. La pente de la régression linéaire entre les données de volume observées et estimées par ces tarifs est largement supérieure à 1. L'EF, plus faible, est différente de la valeur du $R^{2}$ de la régression linéaire. Le RMSE et l'erreur relative sont importants, entre $5,35 \mathrm{~m}^{3}$ et $6,88 \mathrm{~m}^{3}$ et entre $-22,1 \%$ et $7,5 \%$, respectivement. Par ailleurs, outre les deux arbres particuliers susmentionnés, les erreurs d'estimation ont tendance à augmenter avec la taille de l'arbre (figure 3b-e, g, m et o-p).

En ce qui concerne Erythrophleum suaveolens, les estimations de volume du modèle M4, de l'équation de l'administration établie pour $E$. ivorense dans la phase III de l'inventaire et de l'équation développée au Gabon, ne sont pas biaisées (tableau V). La pente de la régression linéaire entre les données de volume observées et estimées par ces tarifs est proche de 1 bien que l'ordonnée à l'origine soit différente de 0 . L'EF est importante et proche de la valeur du $R^{2}$ de la régression linéaire pour ces tarifs. Le RMSE et l'erreur relative sont faibles, entre 1,81 et $2,26 \mathrm{~m}^{3}$, et entre 0,1 et
- 13,3\%, respectivement. Les autres équations de l'administration et l'équation développée à M'Baïki (Rca) sousestiment significativement le volume. La pente de la régression linéaire entre les données de volume observées et estimées par ces tarifs est largement supérieure à 1. L'EF, plus faible, est différente de la valeur du $R^{2}$ de la régression linéaire. Le RMSE et l'erreur relative sont plus importants, entre $2,07 \mathrm{~m}^{3}$ et $4,27 \mathrm{~m}^{3}$, et entre $-12,5 \%$ et $-29,9 \%$, respectivement. Les erreurs d'estimation ont tendance à augmenter avec la taille de l'arbre (figure 4b-c, e-g et i).

Quant à Pericopsis elata, seules les estimations de volume du modèle M4 ne sont pas biaisées (tableau V). La pente de la régression linéaire entre les données de volume observées et estimées par ce tarif est proche de 1 et l'ordonnée à l'origine est proche de 0 . L'EF est importante et proche de la valeur du $R^{2}$ de la régression linéaire. Le RMSE et l'erreur relative sont faibles, $1,15 \mathrm{~m}^{3}$ et 4,7\%, respectivement. Les équations de l'administration et l'équation développée au Ghana sous-estiment significativement le volume. La pente de la régression linéaire entre les données de volume observées et estimées par ces tarifs est largement supérieure à 1 . L'EF, plus faible, est différente de la valeur du $R^{2}$ de la régression linéaire. Le RMSE et l'erreur relative sont plus importants, entre $1,66 \mathrm{~m}^{3}$ et $2,94 \mathrm{~m}^{3}$, et entre $-11,1 \%$ et $-43,6 \%$, respectivement. Les erreurs d'estimation ont tendance à augmenter avec la taille de l'arbre (figure 5b-e). 

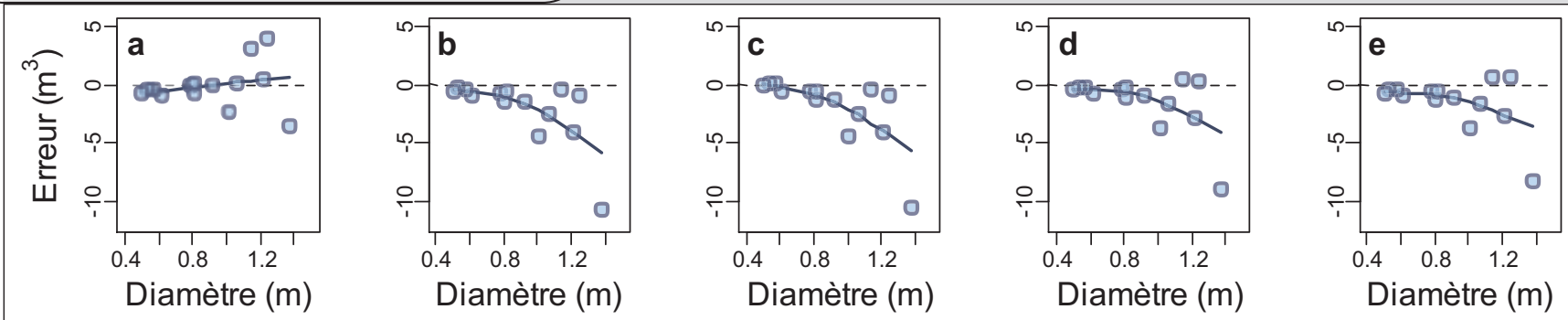

Figure 4.

Erythrophleum suaveolens : erreur d'estimation du tarif de cubage établi dans le cadre de cette étude (a) et des équations utilisées par l'administration camerounaise (b-e pour les phases d'inventaire I-IV) pour Erythrophleum suaveolens en fonction du diamètre (en $\mathrm{m}$ ). La courbe correspond à l'ajustement de la régression non paramétrique. Une valeur nulle indique une adéquation parfaite entre l'estimation du volume et le volume obtenu par la méthode des billons successifs. Les valeurs négatives et positives indiquent les sous- et sur estimations, respectivement.
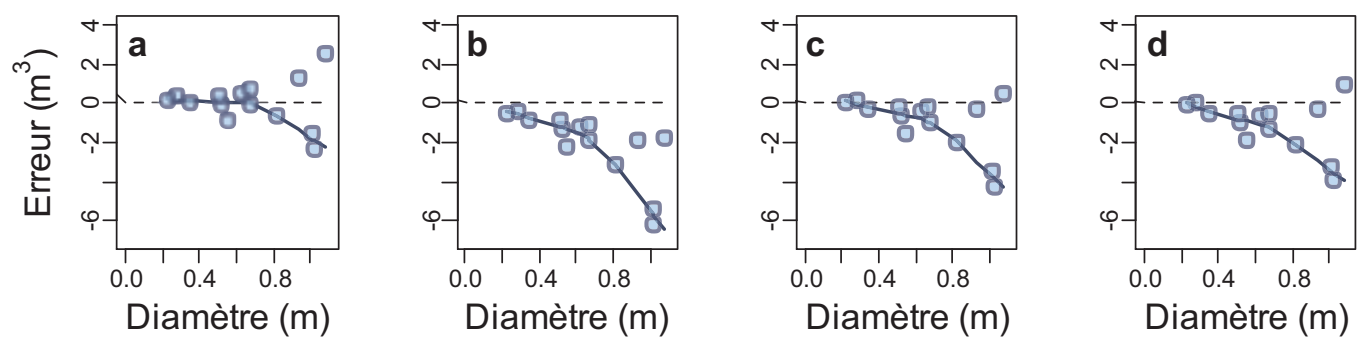

Figure 5.

Pericopsis elata : erreur d'estimation du tarif de cubage établi dans le cadre de cette étude (a) et des équations utilisées par l'administration camerounaise (b-d pour les phases II-IV) pour Pericopsis elata en fonction du diamètre (en m). La courbe correspond à l'ajustement de la régression non paramétrique. Une valeur nulle indique une adéquation parfaite entre l'estimation du volume et le volume obtenu par la méthode des billons successifs. Les valeurs négatives et positives indiquent les sous- et surestimations, respectivement.

\section{Discussion et recommandations}

\section{De nouveaux tarifs de cubage}

Les tarifs de cubage présentés dans cette étude ont été établis à partir de données dendrométriques obtenues par échantillonnage destructif de 43 arbres. L'effectif échantillonné est relativement restreint par rapport aux recommandations usuelles (entre 30 et 100 arbres pour un peuplement homogène ; RONDEUX, 1999), et à des études similaires en forêt tempérée où l'effectif peut atteindre 5000 et 7000 arbres respectivement pour Fraxinus excelsior en Suède et Picea abies en Norvège (ZIANIS et al., 2005). Il faut cependant noter que, dans cette étude, une attention particulière a été portée pour avoir une répartition uniforme des diamètres de l'échantillon et ce jusque 1,93 m de diamètre pour Entandrophragma cylindricum, 1,38 m pour Erythrophleum suaveolens, et 1,08 m pour Pericopsis elata. Parmi les quatre types de modèle de cubage testés, le modèle non linéaire a été retenu pour les trois espèces étudiées. Les équations de volume retenues sont les suivantes, $V=12,724 \times D^{2,000}$ pour $E$. cylindricum, $V=10,920 \times D^{2,248}$ pour $E$. suaveolens, et $V=12,883 \times D^{2,157}$ pour $P$. elata, où le volume $V$ et le diamètre $D$ sont exprimés en $\mathrm{m}^{3}$ et en $\mathrm{m}$, respectivement. On peut remarquer des différences entre les trois essences sur la valeur des paramètres, outre le fait que $E$. cylindricum peut atteindre des diamètres bien plus importants que $E$. suaveolens et $P$. elata. Les tarifs développés dans le cadre de la présente étude pourraient être utilisés à l'avenir pour estimer le volume des arbres dont le dia- mètre est compris dans l'intervalle ayant servi à construire ces tarifs, soit 0,3-2,0 m, 0,5-1,4 m et 0,2-1,1 m pour $E$. cylindricum, $E$. suaveolens et $P$. elata, respectivement, et dans une zone dont les conditions écologiques sont proches de celle de notre zone d'étude. Cette zone correspondrait à celle couverte par les forêts semi-décidues (LETOUZEY, 1968) sur sol ferralitique (MARTIN, SEGALEN, 1966). Avant toute utilisation, il faudrait néanmoins valider ces nouveaux tarifs sur un échantillon de validation différent de celui qui a été utilisé pour la calibration.

Toutefois, pour E. cylindricum, d'autres tarifs pourraient également être utilisés. En effet, cette étude a montré que diverses équations développées en Afrique fournissaient des estimations non biaisées du volume d'E. cylindricum, et ce sur l'ensemble de la gamme de diamètres. Parmi ces équations, il y a des tarifs de cubage linéaires : les équations du projet Api à Dimako (Cameroun), du Mayombe (Congo), de Côte d'Ivoire (y compris Mopri) et du Ghana. Il y a également deux tarifs de cubage non linéaires, ceux développés au Gabon et en Rca. Ces tarifs ne sont pas significativement différents des modèles locaux correspondants : il s'agit des modèles $M 2$ et $M 3$ pour les modèles linéaires et du modèle M4 pour les deux modèles non linéaires, puisque les paramètres sont toujours inclus dans l'intervalle de confiance des paramètres locaux. Quand plusieurs tarifs sont disponibles et appropriés pour une essence, se pose alors la question du choix du tarif de cubage pour l'établissement ou la révision du plan d'aménagement (CHEVALIER, PASQUIER, 2011). 
Par ailleurs, bien que de forme différente, ces équations ont néanmoins toutes en commun le fait d'avoir été établies à partir d'un effectif comprenant des arbres de grande taille, allant jusqu'à $1,70 \mathrm{~m}$ de diamètre. D'après ces observations et les résultats de cette étude, il apparaît que la forme du tarif de cubage importe moins que la taille des arbres ayant servi à le construire. En effet, si le modèle non linéaire (M4) a été retenu dans cette étude pour les trois essences, il est possible de noter que les trois modèles linéaires testés se comportaient relativement bien. En République démocratique du Congo, MALIRO et al. (2010) ont récemment montré que les tarifs de cubage pour Gilbertiodendron dewevrei, Guarea thompsonii et Scorodophloeus zenkeri variaient fortement entre espèces, mais étaient relativement cohérents pour une même espèce entre différentes zones géographiques. Il est cependant possible d'envisager l'existence de variations intra-spécifiques de la relation entre le volume et le diamètre des arbres au sein de différentes zones géographiques, résultant d'une combinaison de facteurs environnementaux tels que le climat et le type de sol, ou de facteurs anthropiques tels que les pratiques sylvicoles. Certes, ces variations sont bien connues pour des essences tempérées (ZIANIS et al., 2005), mais, pour les essences tropicales africaines, ces différences sont moins bien établies, à l'exception de certaines espèces clés comme Terminalia superba (fraké) ou Vitellaria paradoxa (karité), qui ont été largement étudiées en forêt naturelle ou en plantation (HENRY et al., 2011). Globalement, en forêt tropicale, il apparaît que le volume du fût et la biomasse aérienne ligneuse, pour un même diamètre, sont plus importants dans les forêts semi-décidues que dans les forêts sempervirentes très humides (BROWN et al., 1989 ; CHAVE et al., 2005 ; HENRY et al., 2011). Ces différences s'expliqueraient par le fait que les sols sont plus acides et moins profonds dans les forêts sempervirentes. II importe donc de définir précisément la zone géographique de validité des tarifs de cubage. Pourtant, dans le cas du Cameroun, la définition des phases de l'inventaire national des ressources forestières (figure 1) n'est pas à même de répondre à cet impératif, étant donné la non-adéquation de ces phases avec la répartition spatiale des types de végétation (LETOUZEY, 1985) et de sol (MARTIN, SEGALEN, 1966).

\section{Une nécessaire révision des équations utilisées par l'administration}

Grâce à cette étude, il a été possible de montrer que les équations utilisées par l'administration forestière implémentées dans le logiciel Tiama sous-estimaient significativement le volume des fûts pour les trois essences étudiées et pour la zone d'étude. Par ailleurs, les erreurs d'estimation des équations utilisées par l'administration sont d'autant plus grandes que la taille de l'arbre augmente. Ces résultats sont difficilement explicables, étant donné qu'aucune métadonnée n'est disponible. Les seules informations accessibles ont été les normes ayant guidé l'échantillonnage et la méthodologie de cubage utilisée (ONADEF, 1992). Celles-ci préconisent une méthodologie de billonnage fictif du tronc de l'arbre sur pied en billons successifs de longueur variable à l'aide d'un relascope et le calcul du volume correspondant à partir de la formule apparentée (DORISCA et al., 2011). En forêt dense, les conditions d'accès et de visibilité sont particulièrement défavorables à l'utilisation du relascope, avec des difficultés de décomposer le tronc en billons plus courts et réguliers et aussi d'appréciation de la coïncidence des fractions de bandes du relascope couvrant le tronc de l'arbre (LANLY, 1965 ; RONDEUX, 1999), ce qui entrave la prise de mesures et limite la précision. En outre, LANLY (1965), dans une publication sur les tarifs de cubage du sapelli et du kosipo en Rca, évoquait déjà l'erreur de sous-estimation du volume réel des billons donnée par la formule du relascope. Ces sources d'erreur pourraient être à l'origine de la sous-estimation des équations de cubage en vigueur au Cameroun. L'estimation du volume étant un outil essentiel pour l'aménagement et la gestion des forêts, il apparaît nécessaire de réviser les tarifs de cubage pour les espèces commercialisables. En effet, cette sous-estimation des volumes conduit de fait à une inadéquation entre les volumes commerciaux calculés lors de la planification de l'exploitation et transmis à l'administration dans le Plan annuel d'opération (Pao) et les volumes effectivement exploités calculés sur parcs. D'après les travaux de TCHATAT et al. (2008) et les informations recueillies auprès du secteur forestier, l'écart entre ces volumes est systématique et la plupart des sociétés forestières ne peuvent exploiter qu'une partie des effectifs prévus dans le Pao, puisque les volumes autorisés sont atteints avec un nombre de tiges bien inférieur à celui qui est prévu.

Une révision des tarifs de cubage au Cameroun apparaît également fondamentale dans le contexte international actuel. Il s'avère que l'estimation précise des volumes est une étape indispensable du processus de légalité Flegt (Forest Law Enforcement, Governance and Trade) auquel a adhéré le Cameroun. Par ailleurs, de nouveaux enjeux émergent dans le cadre de la mise en œuvre du mécanisme de Réduction des émissions dues à la déforestation et à la dégradation des forêts (Redd+). Parmi les outils conseillés par le Groupe d'experts intergouvernemental sur l'évolution du climat (Giec), les tarifs de cubage combinés à des facteurs d'expansion sont utilisés pour estimer la biomasse des arbres à partir des données de diamètre dans le deuxième « Tier » méthodologique (AALDE et al., 2006). Les pays d'Afrique centrale étant parmi les plus engagés dans la mise en œuvre d'une stratégie nationale Redd+, il est crucial de disposer d'outils performants d'estimation du volume et de la biomasse des arbres.

La méthodologie nécessaire à la révision des tarifs de cubage au Cameroun devrait s'appuyer sur les résultats de cette étude, sur des ouvrages de référence pour les forêts tempérées (RONDEUX, 1999) et sur la publication récente d'un manuel de construction des équations de volume et de biomasse des arbres, intégrant les mesures de terrain et le traitement des données (PICARD et al., 2012). Au préalable, il est indispensable de définir des zones écologiques homogènes reposant sur la prise en compte des données phytogéographiques, climatiques, géologiques et pédologiques. Cette étape est un passage obligé pour définir la zone d'application de tarifs de cubage techniquement robustes. 


\section{Remerciements}

L'étude a été réalisée dans le cadre du projet d’Estimation de la biomasse aérienne ligneuse en Afrique centrale (Ebalac) financé par l'Unité Gestion des ressources forestières et des milieux naturels de Gembloux Agro-Bio Tech, Université de Liège. Les auteurs remercient la société Pallisco et l'association sans but lucratif Nature+ pour l'appui logistique dans la récolte des données, Jacques Hébert et Yves Brostaux pour leur appui dans la mise en place du protocole expérimental et leurs conseils dans l'analyse des données.

\section{Références bibliographiques}

AALDE H., GONZALEZ P., GYTARSKY M., KRUG T., KURZ W. A., OGLE S., RAISON J., SCHOENE D., RAVINDRANATH N. H., ELHASSAN N. G., 2006. Forest Land. In: Agriculture, Forestry and Other Land Use. Volume 4 of the IPCC Guidelines for National Greenhouse Gas Inventories. Hayama, Japon, Institute for Global Environmental Strategies (IGES), 83 p.

AMSALLEM I., KONE P. D., WILKIE M. L., NGANDJI M., 2004. Gestion forestière en Afrique centrale : à la recherche de l'excellence. Bois et Forêts des Tropiques, 281 : 5-18.

BOURLAND N., KOUADIO L., LEJEUNE P., SONKÉ B., PHILIPPART J., DAÏNOU K., FÉTÉKÉ R. F., DOUCET J.-L., 2012a. Ecology of Pericopsis elata (Fabaceae) an Endangered Timber Species in Southeastern Cameroon. Biotropica, 44 (6): 840-847.

BOURLAND N., KOUADIO Y. L., FÉTÉKÉ F., LEJEUNE P., DOUCET J.L., 2012b. Ecology and management of Pericopsis elata (Harms) Meeuwen (Fabaceae) populations: a review. Biotechnologie, Agronomie, Société et Environnement, 16 (4) : 486-498.

BROWN S., GILLESPIE A. J. R., LUGO A. E., 1989. BiomasS estimation methods for tropical forests with applications to forest inventory data. Forest science, 35 (4): 881-902.

CAILLIEZ F., 1980. Volume estimation. In: Forest volume estimation and yield prediction. Rome, Italie, Fao, 98 p.

CHAVE J., ANDALO C., BROWN S., CAIRNS M., CHAMBERS J., EAMUS D., FÖLSTER H., FROMARD F., HIGUCHI N., KIRA T., LESCURE J.-P., NELSON B. W., OGAWA H., PUIG H., RIÉRA B., YAMAKURA T., 2005. Tree allometry and improved estimation of carbon stocks and balance in tropical forests. Fcologia, 145 (1): 87-99.

CHEVALIER J.-F., PASQUIER A., 2011. Après dix années de gestion, déroulement et enseignements issus de la révision d'un plan d'aménagement forestier en République centrafricaine. Bois et Forêts des Tropiques, 310 : 25-41.

DORISCA S., DURRIEU DE MADRON L., FONTEZ B., GIRAUD A., RIÉRA B., 2011. Établissement d'équations entre le diamètre et le volume total de bois des arbres, adaptées au Cameroun. Bois et Forêts des Tropiques, 308 : 87-94.
DUMINIL J., HEUERTZ M., DOUCET J.-L., BOURLAND N., CRUAUD C., GAVORY F., DOUMENGE C., NAVASCUÉS M., HARDY O. J., 2010. CpDNA based species identification and phylogeography: application to African tropical tree species. Molecular Ecology, 19 (24): 5469-5483.

FAO, 2007. Évaluation des ressources forestières nationales du Cameroun 2003-2004. Yaoundé, Cameroun, Ministère des Forêts et de la Faune, 93 p.

GERDAT (CIRAD), 1974. Fiches techniques avec planche couleur : sapelli. Bois et Forêts des Tropiques, 154 : 27-40.

GERDAT (CIRAD), 1977. Fiches techniques : tali. Bois et Forêts des Tropiques, 176 : 17-31.

HAWTHORNE W. D., 1995. Ecological profiles of Ghanaian forest trees. Oxford, Royaume-Uni, Université d'Oxford, 345 p.

HENRY M., BOMBELLI A., TROTTA C., ALESSANDRINI A., BIRIGAZZI L., SOLA G., VIEILLEDENT G., SANTENOISE P., LONGUETAUD F., VALENTINI R., 2013. GlobAllomeTree: international platform for tree allometric equations to support volume, biomass and carbon assessment. iForest-Biogeosciences and Forestry, 6 (1): e1-e5.

HENRY M., PICARD N., TROTTA C., MANLAY R. J., VALENTINI R., BERNOUX M., SAINT-ANDRÉ L., 2011. Estimating tree biomass of sub-Saharan African forests: a review of available allometric equations. Silva Fennica, 45 (3): 477-569.

KOUADIO Y. L., 2009. Mesures sylvicoles en vue d'améliorer la gestion des populations d'essences forestières commerciales de l'Est du Cameroun. Thèse, Faculté universitaire des sciences agronomiques, Gembloux, Belgique, 241 p. + annexes.

KOUADIO Y. L., DOUCET J.-L., 2009. Étude du comportement de Baillonella toxisperma Pierre (moabi) dans les trouées d'abattage enrichies. Biotechnologie, Agronomie, Société et Environnement, 13 (2) : 317-324.

LANLY J.-P., 1965. Les tarifs de cubage. Bois et Forêts des Tropiques, $100: 19-35$.

LETOUZEY R., 1968. Étude phytogéographique du Cameroun. Lechevalier, France, $511 \mathrm{p}$.

LETOUZEY R., 1985. Notice de la carte phytogéographique du Cameroun. Yaoundé, Cameroun, Institut de la recherche agronomique, Toulouse, France, Institut de la carte internationale de la végétation.

MALIRO T. K., DIMANDJA J. P. L., PICARD N., 2010. Volume equations and biomass estimates for three species in tropicalmoist forest in the Orientale province, Democratic Republic of Congo. Southern Forests: a Journal of Forest Science, 72 (3-4): 141-146.

MARTIN D., SEGALEN P., 1966. Notice explicative de la carte pédologique du Cameroun oriental. Yaoundé, Cameroun, Centre Orstom. 
MAYAUX P., BARTHOLOMÉ E., FRITZ S., BELWARD A., 2004. A new land-cover map of Africa for the year 2000. Journal of Biogeography, 31 (6): 861-877.

MAYER D. G., BUTLER D. G., 1993. Statistical validation. Ecological modelling, 68 (1): 21-32.

ONADEF, 1992. Normes d'études d'arbres. Yaoundé, Cameroun, Ministère de l'Environnement et des Forêts, $56 \mathrm{p}$.

PICARD N., SAINT-ANDRÉ L., HENRY M., 2012. Manuel de construction d'équations allométriques pour l'estimation du volume et la biomasse des arbres : de la mesure de terrain à la prédiction. Rome, Italie, Fao, Montpellier, France, Cirad, 222 p.

R DEVELOPMENT CORE TEAM, 2013. R: A language and environment for statistical computing. R Foundation for Statistical Computing.

RONDEUX J., 1999. La mesure des arbres et des peuplements forestiers. Gembloux, Belgique, Les Presses agrono-

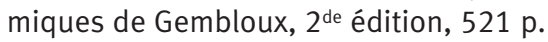

TCHATAT M., NDOUMBÈ NKENG M., ABENA J. C., FOAHOM B., 2008. Volumes de bois autorisés à l'exploitation au Cameroun : détermination des valeurs maximales de dépassement tolérable. Bois et Forêts des Tropiques, 295 : 35-46.

DE WASSEIGE C., DEVERS D., DE MARCKEN P., EBA'A ATYI R., NASI R., MAYAUX P., 2009. Les forêts du Bassin du Congo État des Forêts 2008. Luxembourg, Office des publications de l'Union européenne, $274 \mathrm{p}$.

WHITE F., 1983. The vegetation of Africa: A descriptive memoir to accompany the Unesco/AETFAT/UNSO vegetation map of Africa. Paris, France, Unesco, 356 p.

ZANNE A. E., LOPEZ-GONZALEZ G., COOMES D. A., ILIC J., JANSEN S., LEWIS S. L., MILLER R. B., SWENSON N. G., WIEMANN M. C., CHAVE J., 2009. Global wood density database. Dryad. http://hdl. handle. net/10255/dryad 235.

ZIANIS D., MUUKKONEN P., MÄKIPÄÄ R., MENCUCCINI M., 2005. Biomass and stem volume equations for tree species in Europe. Silva Fennica Monographs 4, 63 p. 